The Avon Longitudinal Study of Parents and Children (ALSPAC) is a transgenerational prospective observational study investigating influences on health and development across the life course. It has collected information on genetic, epigenetic, biological, psychologi$\mathrm{cal}$, social and other environmental exposures in relation to a diverse range of health, social and developmental outcomes. Recruitment sought to enrol pregnant women in the Bristol area of the UK during 1990-92. There were 13761 women (contributing 13867 pregnancies) recruited. This was extended to include additional children eligible using the original enrolment definition up to the age of 18 years. The children from 14541 pregnancies were recruited in 199092, increasing to 15247 pregnancies by the age of 18 years. The resource comprises a wide range of phenotypic and environmental measures in addition to biological samples, genetic and epigenetic information and linkage to health and administrative records. The study is celebrating its $21^{\text {st }}$ Anniversary this year and over 700 peerreviewed articles have been published using data from ALSPAC. The study has made contributions to understanding across a range of disciplines, exposures and outcomes. The presentation will present my view of the key contributions the study has made to date.

\section{REGIONAL DIFFERENCES IN PERI- AND NEONATAL OUTCOMES OF EXTREMELY PRETERM INFANTS IN SWEDEN (EXPRESS)}

doi:10.1136/archdischild-2012-302724.0204

'FH Serenius, ' $\mathrm{G}$ Sjörs, ${ }^{2} \mathrm{M}$ Blennow, ${ }^{3} \mathrm{~V}$ Fellman, ${ }^{4} \mathrm{G}$ Holmström, ${ }^{5} \mathrm{~K}$ Marsal, ${ }^{6} \mathrm{E}$ Lindberg, ${ }^{7} \mathrm{E}$ Olhager, ${ }^{8} \mathrm{~L}$ Stigson, ${ }^{9} \mathrm{M}$ Westgren, ${ }^{10} \mathrm{~K}$ Källen, The EXPRESS Study Group. ${ }^{1}$ Women's and Children's Health, Uppsala University, Uppsala; '2Dept of Pediatrics, Karolinska University Hospital, Huddinge, Stockholm; ' ${ }^{3}$ Dept of Pediatrics, Lund University, Lund; ${ }^{4}$ Dept Ophthalmology, Uppsala University, Uppsala; ${ }^{5}$ Dept of Obstetrics and Gynecology, Lund University, Lund; ' ${ }^{6}$ Dept of Pediatrics, Örebro University, Örebro; 'Dept of Pediatrics, Linköping University, Linköping; ${ }^{8}$ Dept of Pediatrics, Sahlgrenska University Hospital, Göteborg; 'Dept of Obstetrics and Gynecology, Karolinska University Hospital, Huddinge, Stockholm; ${ }^{10}$ Centre for Reproductive Epidemiology, Lund University, Lund, Sweden

Background The EXPRESS study has shown favourable peri-and neonatal outcomes of extremely preterm infants (EPT, <27weeks) in Sweden compared with similar studies.

Objective To determine whether there are differences in peri- and neonatal outcomes in spite of favourable national rates and whether outcomes can be related to regional differences in the use of perinatal interventions.

Methods Population-based prospective study of all EPT children born in Sweden from April1, 2004, to March 31, 2007. Of 1011 births, 707 were born alive and 497 survived to one year. Each region was assigned a perinatal activity score (PAS) based on the rate of selected perinatal interventions. Mortality rates were calculated, adjusted for background factors and related to PAS.

Results There were few regional differences in demographic background data. PAS varied from 74 to 100 (median 82) between regions. When 3 regions with the highest PAS (median 98) were compared with 4 regions with lower PAS (median 79), the following adjusted odds ratios (AOR) were found for infants born at 22-26 weeks: Perinatal death, AOR 0.6 (95\%CL 0.4-0.8), infant mortality $0.6(95 \% \mathrm{Cl} 0.4-0.9)$. There was no increase in the odds for survival with severe neonatal morbidity; AOR 0.7 (95\% Cl 0.5-1.0). When stratified by gestational age, increased survival was confined to infants born at 22-24 weeks. Regional differences were nullified when early deaths ( $<12$ hours) were excluded.

Conclusions There are differences in peri- and neonatal outcomes between regions in Sweden which can be explained by the intensity of perinatal interventions.

205 LUNG FUNCTION IN ADULTS BORN PRETERM - THE HELSINKI STUDY OF VERY LOW BIRTH WEIGHT ADULTS

doi:10.1136/archdischild-2012-302724.0205
'M Sipola-Leppänen, 'HK Saarenpää, 'M Tikanmäki, ${ }^{2,3 P}$ Hovi, ${ }^{2,3} \mathrm{~K}$ Wehkalampi, ${ }^{4} \mathrm{M}$ Siltanen, ${ }^{1,5} \mathrm{M}$ Vääräsmäki, ${ }^{2} \mathrm{AL}$ Järvenpää, ${ }^{3,6} \mathrm{JG}$ Eriksson, ${ }^{2} \mathrm{~S}$ Andersson, ${ }^{2,3} \mathrm{E}$ Kajantie ${ }^{1}$ National Institute for Health and Welfare, Oulu, ${ }^{2}$ Hospital for Children and Adolescents, Helsinki University Central Hospital; ${ }^{3}$ National Institute for Health and Welfare, Helsinki; ${ }^{4}$ Hyvinkää Hospital, Hyvinkää; ${ }^{5}$ Department of Obstetrics and Gynaecology, Oulu University Hospital, Oulu; ${ }^{6}$ Department of General Practice and Primary Health, University of Helsinki, Helsinki, Finland

Backround and aims Adolescents born at very low birth weight $(<1500 \mathrm{~g}, \mathrm{VLBW})$ have higher rates of respiratory symptoms and reduced lung function as compared with those born at term. Only few studies, however, have extended to adult life. We studied the association of preterm birth at VLBW with lung function in young adults.

Methods We used spirometry (Medikro $\left.{ }^{\circledR}\right)$ to measure pulmonary function in 160 VLBW subjects and in 162 term-born controls (mean age 22.5 years) as a part of the Helsinki Study of Very Low Birth Weight Adults. BPD was diagnosed by a clinician based on Northway's criteria.

Results Figure 1 shows the mean values and differences in lung function tests between the groups. Forced expired volume in $1 \mathrm{sec}-$ ond (FEV1), the FEV1/FVC (forced vital capacity) ratio, peak expiratory flow (PEF), maximal expiratory flow at $25 \%$ or $50 \%$ (MEF $25 \%$ and $50 \%$ ) were lower in adults born at VLBW than in those born at term. This finding was strongest in VLBW adults with a history of BPD but was present also in VLBW adults with no history of BPD.

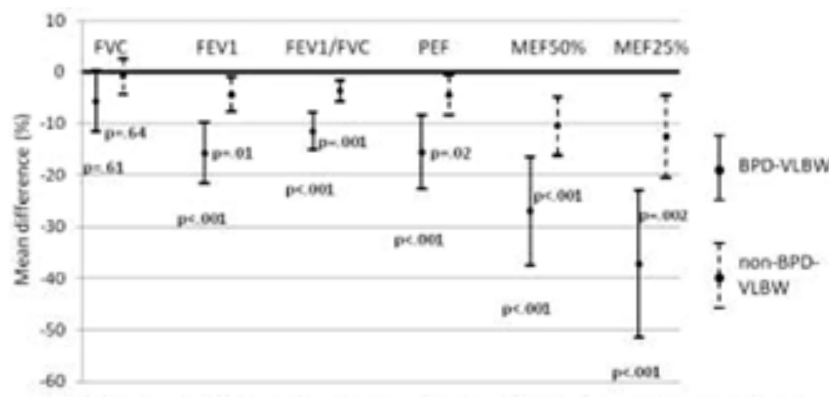

Abstract 205 Figure 1 The mean difference in lung function test between VLBW adults with or without broncopulmonary dysplasia [BPD] (error bars) and term born controls (zeroline) adjusted for age, sex, height, BMI, parental education, maternal smoking during pregnancy, current daily smoking of the subject, and the frequency of leisure-time conditioning physical activity

The mean difference in lung function test.

Conclusions Reduced FEV1/FVC, PEF and $\mathrm{MEF}_{25-50 \%}$ suggest a medium and small airway obstruction among young adults born at VLBW. While this finding is strongest among BPD survivors, it is present also among VLBW adults with no history of BPD. This may be a risk factor for later obstructive pulmonary disease.

\section{NEONATAL INFECTION AND 5-YEAR NEURODEVELOPMENTAL OUTCOMES OF VERY PRETERM INFANTS: THE EPIPAGE STUDY}

doi:10.1136/archdischild-2012-302724.0206

${ }^{1,2} \mathrm{~A}$ Mitha, ${ }^{3} \mathrm{PY}$ Ancel, 1,2P Truffert, ${ }^{3} \mathrm{~L}$ Foix L'Hélias, EPIPAGE Study Group. ${ }^{1} \mathrm{NICU}$, Jeanne de Flandre Hospital; ${ }^{2}$ Faculty of Medicine, Lille-Nord-de-France University, Lille; ${ }^{3}$ Inserm Unit 953, Paris, France

Background and aims To determine if neonatal infections are associated with increased risks of adverse neurodevelopment at 5 years of age in a population-based cohort of very preterm children. Methods We included all live births between 22 and 32 weeks of gestation from 9 regions in France in 1997 (EPIPAGE study). Of the 2665 live-births, 2193 were eligible for follow-up evaluation at 5 years of age, 1769 had a medical examination and 1495 a cognitive 
assessment. Cerebral palsy and cognitive impairment were studied according to early onset sepsis (EOS) and late onset sepsis (LOS) after adjustment for potential confounding variables using multivariate logistic regression models.

Results In total, 139 (5\%) of the 2665 live births included had an EOS alone (without LOS associated), 752 (28\%) a LOS alone (without EOS associated) and 64 (2\%) EOS and LOS associated. At 5 years, the rate of cerebral palsy was 9\% (157/1769) and cognitive impairment $12 \%(177 / 1495)$. Compared with uninfected infants, cerebral palsy was increased in the group of EOS alone $(\mathrm{OR}=1.70$, $95 \% \mathrm{CI}: 0.84-3.45)$, in the group of LOS alone (OR $=1.71,95 \% \mathrm{CI}$ : 1.14-2.56), and this risk was increased further when EOS and LOS were associated (OR $=2.33,95 \% \mathrm{CI}: 1.02-5.33)$. There was no association between neonatal infection and cognitive impairment.

Conclusion Neonatal infections among very preterm infants are associated with an increased risk of cerebral palsy at 5 years of age, particularly when EOS and LOS are cumulative.

\section{MATERNAL FATTY ACIDS INTAKE DURING PREGNANCY AND LATER CHILD COGNITIVE DEVELOPMENT IN THE EDEN MOTHER-CHILD COHORT STUDY}

doi:10.1136/archdischild-2012-302724.0207

1,2JY Bernard, 1,2M de Agostini, 1,2 A Forhan, 3,4V Champion, ${ }^{3,4} \mathrm{M}$ Kaminski, 1,2MA Charles, 1,2B Heude, EDEN Mother-child Cohort Study Group. ' Center for Research in Epidemiology and Population Health, Inserm, Villejuif; ${ }^{2}$ Faculté de Médecine, Université Paris-Sud 11, Le Kremlin-Bicêtre; ${ }^{3} E$ pidemiological Research Unit on Perinatal Health and Women's and Children's Health, Inserm Unit 953; ' Université Pierre et Marie Curie, Université Paris 6, Paris, France

Background and aims Polyunsaturated Fatty Acids (PUFA) are needed for child brain development, especially $n$-3 PUFAs. Prenatal exposure depends on maternal lipids intake during pregnancy. We aimed to investigate associations between maternal PUFAs intake during pregnancy and later child cognitive development.

Methods In 1066 children of the EDEN mother-child cohort, we assessed cognitive development at 3 years with the Ages and Stages Questionnaire (ASQ, score between 0 and 300). Maternal lipids intake during pregnancy was evaluated after delivery, using a food frequency questionnaire and a food-composition table. We investigated associations between PUFAs intake and ASQ score using multiple linear regressions adjusted for centre, child's age, gender and gestational age, maternal tobacco and alcohol consumptions, parental education, siblings, caregivers and preschool attendance.

Results Mean ASQ score was 270.1 ( \pm 29.4$), n-6 / n-3$ ratio in food intake was $10.0( \pm 2.3)$ and total $n-3$ PUFAs intake was $0.47 \%( \pm 0.09)$ of total energy intake. In crude analyzes, ASQ score was positively associated with each three $n$-3 PUFAs ( $\alpha$-linolenic, eicosapentaenoic and docosapentaenoic acids) and negatively with linoleic acid and $n-6 / n-3$ ratio. After adjustment, ASQ score remained significantly associated with $n-6 / n-3$ ratio $(\beta=-1.16$; $S E=0.37 ; P=0.0015)$. Association with total $n-3$ PUFAs tended to persist $(\beta=1834$; $S E=985$; $P=0.063$ )

Conclusions After adjustment for confounders, especially maternal education, higher $n$-3 PUFAs intake and thus lower $n-6 / n-3$ ratio in pregnancy food consumption were associated with better cognitive development in early childhood. We observed similar results with prepregnancy lipids intake. Our study suggests a role of prenatal nutrition on childhood cognitive development.

\section{MANAGEMENT OF NEWBORNS WITH SUSPECTED OR PROVEN CONGENITAL TOXOPLASMA INFECTION IN THE FIRST TEN DAYS OF LIFE}

doi:10.1136/archdischild-2012-302724.0208

P Garcia-Meric. Université de la Mditerranée, Marseille, France
Congenital toxoplasmosis is caused by transplacental fetal contamination following maternal primary infection.

Risk of transmission increases with gestational age. Severity is higher when transmission occurs before 20 WG leading to abortion, fetal loss, prematurity or severe fetal damage (particulary neurological). After this period, infection mainly affects the eye.

Since establishment of prenatal screening in Austria and France, toxoplasmosis has declined. Early maternal treatment (spiramycin or pyrimethamine-sulfonamide) has shown a lower incidence of fetal sequelae. Combination of ultrasound follow-up, fetal MRI, real time PCR on amniotic fluid allows antenatal diagnosis of severe forms of CT in which termination of pregnancy are accepted. Only $15 \%$ of infected liveborn children have clinical signs.

In countries with high incidence of CT and without national program, prenatal and/or neonatal screening are required to improve medical care and minimize sequelae (chorioretinitis, hydrocephalus ...). Low prevalence countries recommand neonatal screening or prevention rules.

In case of prenatal diagnosis, clinical examination, serological tests (detection of specific IgM/IgA confirmed at day 10, comparaison of immunologic mother- child profiles), transfontanellar ultrasonography and ocular fundus are performed at birth. In lack of prenatal screening, when children are symptomatic, maternal serology (with avidity test), PCR on placenta, neonatal lumbar punction and tomography are added. In asymptomatic children, diagnosis will be evocated when complications appear (visual impairment, psychomotor delay, seizures...).

Serological tests should be interpreted cautionnely in early or late maternal infection, maternal treatment without amniocentesis. Thus infected children have to be treated early to reduce risk of chorioretinitis.

\section{MANAGEMENT OF CHILDREN WITH SUSPECTED OR PROVEN CONGENITAL TOXOPLASMOSIS FROM DAY 10 TO THE END OF THEIR FIRST YEAR}

doi:10.1136/archdischild-2012-302724.0209

M Wallon. Service de Parasitologie, Hopital de la Croix-Rousse, Hospices Civils de Lyon, Lyon, France

Management from Day 10 to Day 365 has two goals according to the results of the work-up performed at birth, and eventually in utero. The first is to start treatment and surveillance in infected newborns and, the second, applying to settings where prenatal screening is performed, is to confirm the absence of infection in newborns who are born from a mother who seroconverted during pregnancy but who show no signs of infection at birth.

When congenital infection is proven the standard attitude in France is to start treatment without delay even newborns with no clinical signs. Treatment relies on a combination of pyrimethamine and sulfonamides but there is no consensus on the type of sulfonamides, on the dosages and rhythm of administration and on the length of treatment, ranging from 3 to 24 months. Children under treatment should be monitored regularly for side effects. The decrease of IgG under treatment is a normal evolution and should not be interpreted as a sign of non-infection. Regular neurological and ophthalmological examinations in the first year of life are also important to detect any signs that would deserve special attention.

In the second case, the absence of clinical and biological signs in utero or at birth significantly decreases the probability of infection. Repeated serological tests remain however necessary to fully exclude infection by monitoring the decrease of $\operatorname{Ig} G$ to undetectable levels. Any neosynthesis of IgG would indicate that the child is infected and warrant starting the same treatment as in infected infants. 